\title{
Effective potentials in a bidimensional vibrated granular gas
}

\author{
Stephanie Velázquez-Pérez ${ }^{1}$, Gabriel Pérez-Ángel ${ }^{2}$ and Yuri Nahmad-Molinari ${ }^{1}$ \\ ${ }^{1}$ Instituto de Física "Manuel Sandoval Vallarta", \\ Universidad Autónoma de San Luis Potosí, \\ Álvaro Obregón 64, San Luis Potosí, SLP, México; \\ ${ }^{2}$ Departamento de Física Aplicada, Centro de Investigación \\ y de Estudios Avanzados del IPN, Unidad Mérida, \\ AP 73 "Cordemex", 97310 Mérida, Yuc., México
}

(Dated: September 18, 2018)

\begin{abstract}
We present a numerical study of the spatial correlations of a quasi-two-dimensional granular fluid kept in a non-static steady state via vertical shaking. The simulations explore a wide range of vertical accelerations, restitution coefficients and packing fractions, always staying below the crystallization limit. From the simulations we obtain the relevant Pair Distribution Functions (PDFs), and effective potentials for the interparticle interaction are extracted from these PDFs via the Ornstein-Zernike equation with the Percus-Yevick closure. The correlations in the granular structures originating from these effective potentials are checked against the originating PDF using standard Monte Carlo simulations, and we find in general an excellent agreement. The resulting effective potentials show an increase of the spatial correlation at contact with the decreasing values of the restitution coefficient, and a tendency of the potentials to display deeper wells for more dissipative dynamics. A general exception to this trend appears for a range of values of the forcing, which depends on the restitution coefficient, but not on the density, where resonant bouncing increases correlations, resulting in deeper potential wells. The nature of these resonances is explored, and shown to be the result of synchronization in the parabolic flights of the particles.
\end{abstract}

PACS numbers: 45.70.-n, 61.20.Ne 


\section{INTRODUCTION}

Granular materials are constituted by macroscopic rigid objects that are too big to undergo Brownian motion, and that dissipate energy when they collide [1, 2]. Therefore, a granular assembly in the presence of gravity tends rapidly to a static configurations, where all the material simply forms a pile on the bottom of whichever container holds it. However, using a continuous injection of energy, it is possible to maintain a granulate in a non-static configuration. The energy is usually injected through vertically shaking the system, using a sinusoidal displacement. In such a case, the motion's amplitude times the square of the angular frequency gives the maximal acceleration of the exciting system, and this value divided by the gravitational acceleration is called its non-dimensional acceleration $\Gamma$. Due to the permanent flow of energy in this kind of granular aggregate, one may observe the appearance of self-organization, allowing for some very interesting phenomena such as oscillons, and a wide variety of beautiful patterns [3]. But it is also possible, with this permanent provision of energy, to obtain statistically stationary configurations containing just a few homogeneous phases. Under these conditions, granular materials may resemble gases, liquids [4] or crystals in equilibrium; and in fact these systems have been used as models for atomic, molecular, and colloidal ensembles in thermal equilibrium [2, 5].

Thus, condensed phases resembling liquids or solids, both crystalline and glassy, have been reported in granular materials under vertical shaking excitation. Their stationary structures have been described [6, 7], and the jammed-like system relaxation dynamics that they display has been reported [8]. In particular, two-dimensional crystalline configurations with hexagonal order [9, 10] (and, with the appropriate confinement, square order [11, 12]), have been found.

These crystalline clusters have been obtained in the laboratory either by increasing the packing fraction, that is, the total volume (area in 2D systems) occupied by the particles, divided by the volume (area) of the container; or by reducing the amplitude of the driving. In the first case the process is basically equal to the crystallization of hard disks in equilibrium [13, 14]: as density is increased the aggregate displays a transition from a fluid to an hexagonal crystalline phase, presumably going through a narrow hexatic phase [8, 13], a behavior similar in all ways to the well-known entropicaly-driven crystallization of monodisperse hard disks [15, 16]. But surprisingly, the formation of hexagonal crystals has been also observed, 
both experimentally and numerically, for values of $\Gamma \approx 1,[9,17,18]$, at very low packing fractions $(\phi=0.42$ in the experiment vs. $\phi \approx 0.71$ for equilibrium crystallization in a gas of hard disks). This crystallization gives an extreme example of the anomalous behavior of vibrated dissipative monolayers, in both their morphology and dynamics, but the differences with a two-dimensional equilibrium gas are not exclusive to condensate phases. One particular issue of interest in these systems is the evolution of density fluctuations, including the creation of large dense clusters [19], a situation that resembles the density fluctuations in a gas approaching a gas-liquid transition.

The existence of effective potentials related to the physical parameters of the shaken granulate, especially to its restitution coefficient, has been proposed in [20], in the spirit of this analogy to the gas-liquid transition, and also to the gas-crystal transition mentioned before. It has been shown in that work how the structure of a given granulate, described by its Pair Distribution Function (PDF) $g(r)$ can be related to an attractive potential, and how the naive expectation of more dissipation implying a more attractive potential does get realized. This idea of introducing effective potentials in a granular medium — dissipative and out of equilibrium as it is-, is an extension of the previous generalizations of interparticle potentials: for instance, the well known Dejaurmin, Landau, Vervey and Overbeek (DLVO) potential [21 23] for colloids with electrostatic interactions, and the depletion interactions due to entropic effects, modeled originally by Asakura and Oosawa [24, 25].

In extending these ideas to vibrated granular matter one simply wants to know whether it is possible to represent the structure of a stationary state as if it were an equilibrium state of a well defined thermodynamic system. Besides the monodisperse quasi-2D granular gas mentioned before [20], that we continue exploring here, this approach has also been tried in the case of a two-species granular gas with horizontal shaking, whose dynamics has been interpreted in terms of a depletion interaction system [26]. The interaction of particles trough a potential well implies a conservative system in which the energy associated to the relative position of two particles is always balanced by their total kinetic energy, producing a characteristic structure, that is, characteristic spatial distribution and correlations. What we propose here is that a set of particles interacting trough dissipative collisions which uniformly receives energy from an oscillating support could, in general, behave effectively as a conservative system, in the sense of statistically reproducing the characteristic spatial distribution of particles produced by the last. 
In this paper we present a computational study (molecular dynamics and Monte Carlo simulations) of a quasi-2D gas of spheres that collide inelastically, excited via vertical shaking. We intend to show that the homogeneous steady state of this system may be described by means of an analogy with equilibrium liquid theory. This is done by means of performing molecular dynamics simulations, getting the corresponding pair distribution functions, and obtaining from them — using the Ornstein-Zernike equation with the Percus-Yevick closure - the associated effective potentials. Furthermore, in order to test the correctness of this analogy, we performed Monte Carlo simulations of hard discs interacting through the effective potentials obtained by the aforementioned molecular dynamic simulations and the simple liquid theory, and compare their respective pair correlation functions as a check of the correctness of the approach.

\section{SIMULATION SCHEME}

We simulate a system quite similar to the usual experimental set-ups that have been used in many studies of granular gases [9, 10]. A monodisperse granulate composed of $N=2000$ hard spheres of diameter $\sigma=0.5 \mathrm{~cm}$ is confined by two horizontal flat interfaces ("floor" and "ceiling") perpendicular to the direction of gravity, separated by a distance $h=3, \sigma=1.5$ $\mathrm{cm}$, making sure in all cases that in the stationary state the grains never reach the ceiling. As an exception, for some control simulations conducted without dissipation, the vertical spacing was set at $h=1.6, \sigma=0.8 \mathrm{~cm}$. The two-dimensional packing fraction is defined by the total projected area covered by particles divided by the simulation cell area, that is, $\phi=N \pi \sigma^{2} /\left(4 L^{2}\right)$. The horizontal cell is a square whose side is adjusted depending on the desired packing fraction, and where periodic boundary conditions are used in the horizontal directions. A sketch of the system is presented in top and lateral views in Fig. (1).

The assembly is sinusoidally vibrated in the vertical direction (parallel to gravity), defining the position of the two horizontal plates as $z_{b}=A \sin (2 \pi f t)$ for the floor and $z_{t}=z_{b}+h$ for the ceiling; here $A$ is the amplitude and $f$ is the frequency of the shaking. The simulations are conducted with 7 different values of the restitution coefficient, given by $\epsilon=0.40$, $0.50,0.60,0.70,0.80,0.90$ and 1.0 , this last one used for the non-dissipative test. We also use four different values of the packing fraction, given by $\phi=0.20,0.30,0.40$ and 0.50 , and at least 5 values of the non-dimensional vertical acceleration $\Gamma$, defined, as mentioned 


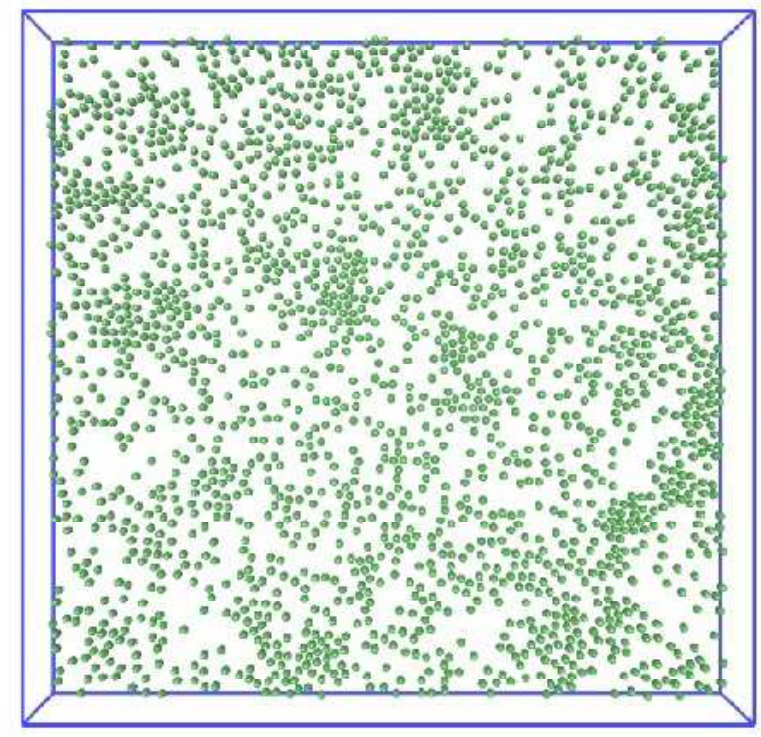

a) Top view.

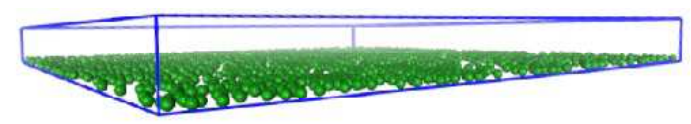

b) Lateral view.

FIG. 1. Scheme of the simulated system in a) Top view, and b) Lateral view. 2000 spheres are vertically shaken by a vertical sinusoidal motion of the containing cell.

before, by $\Gamma=(2 \pi f)^{2} \mathrm{~A} / \mathrm{g}$, and given by $\Gamma=1.05,1.15,1.25,1.35$ and 1.45 for each case. For the study of resonances, to be discussed latter, we also used a much finer sweep over $\Gamma$. The gravitational acceleration is $g=9.81 \mathrm{~m} / \mathrm{s}^{2}$, the frequency is kept fixed at $f=70 \mathrm{~Hz}$, and the tangential friction coefficient is fixed at $\mu=0.25$ (again, with the exception of the control simulation that has $\mu=0$ ). Notice that it is possible to scale out some magnitudes, but we prefer to use real physical quantities; it should be understood that the reported results can be scaled to other sets of parameters, making sure that the process is such that $g$ keeps its numerical value. The bounds in the ranges of parameters explored are set by the need to keep some of the basic characteristics of the system; in particular, larger values of $\Gamma$ allow sometimes a grain to jump over another, and larger values of $\phi$ begin to introduce crystallization. 
The simulations are conducted using an Event-Driven Molecular Dynamics (EDMD) algorithm [27, 28]. In this algorithm, one calculates analytically the trajectory in space of all spheres, and finds the times for all possible future collisions. These collision times are sorted, and the system is then ballistically advanced to the time of the first collision. This collision is then realized, preserving linear momentum and angular momentum around the point of contact, but not energy. Immediately after, the list of future collisions is updated, then sorted, and the system is again advanced ballistically to the next collision. Dissipation arises due to two effects: as seen in the center of mass, the particles separate with normal velocities which are only a fraction $\epsilon$ of the incident normal velocities, making the collision viscoelastic; also, the tangential velocities are reduced by a tangential restitution coefficient $\epsilon_{s}$, which depends on the friction coefficient $\mu$, keeping the conservative limit $\epsilon_{s}=1$ for $\mu=0$. Both dissipative processes have also an effect on the rotations of the particles. The full equations used to solve the collision are given in [29, 30]. A couple of extra points should be mentioned: first: collision times between spheres can be calculated exactly even in the presence of gravity, in the same way that they can be solved without gravity, just solving a quadratic equation. Second: solving for the time of collision between a particle falling in a parabola and the floor (or the ceiling) moving sinusoidally requires a numerical scheme. Here we have implemented root-finding routine specifically designed to calculate this type of intersection.

It is understood that we always use $\Gamma>1$, to avoid situations where a grain no longer does finite length bounces against either other grain or a wall. Such a situation forces an EDMD code to perform an infinite number of collisions in a finite time, that is, to undergo inelastic collapse [31]. Some remedies can be applied, such as to take the restitution coefficient $\epsilon$ equal to 1 for very small relative velocities [32], or to ignore collisions that are less than some small $\Delta t$ apart, and even freezing the dynamics of very dense clusters [33]. But any way one looks at it, ED is unable to deal with non-bouncing grains. This issue is avoided for $\Gamma>1$. 


\section{RESULTS: PAIR DISTRIBUTION FUNCTIONS AND EFFECTIVE POTEN-}

\section{TIALS}

For our purposes, the main results from the simulations mentioned above are their PDFs, which are extracted from a sampling of configurations using well known methods. These PDFs are then assumed to originate in an equilibrium 2D fluid, and to be determined, therefore, by an interparticle potential, along with temperature and density. However, the relationship between these potentials and the corresponding PDFs is not easily resolved. We will use here the homogeneous Ornstein-Zernike (OZ) equation

$$
h\left(r_{12}\right)=c\left(r_{12}\right)+\rho \int \mathrm{d}^{3} \mathbf{r}_{3} c\left(r_{13}\right) h\left(r_{32}\right)
$$

where $r_{i j}=\left|\mathbf{r}_{i}-\mathbf{r}_{j}\right|, \rho$ is the number particle density, and $h(r) \equiv g(r)-1$ and $c(r)$ are the total correlation function and the direct correlation function, respectively. In practice, $c(r)$ is actually defined by the OZ equation itself [34]. The relation between these two correlation functions can be written in a much simpler form using Fourier transform, where one finds

$$
\hat{h}(k)=\frac{\hat{c}(k)}{1-\rho \hat{c}(k)} .
$$

Still, in the absence of an independent relationship, this equation is of little use. For hard spheres — and, in general, short range potentials, in either dense or diluted situations - , a good closure relationship is given by the Percus-Yevick (PY) approximation [35]

$$
c(r)=[h(r)+1]\left[1-\exp \left(u(r) / k_{B} T\right)\right] .
$$

With this approximation one can then generate a value for the quotient $u(r) / k_{B} T$, once the

$g(r)$ and $\rho$ are given. This requires: the evaluation of $\hat{h}(k)$, which in $2 \mathrm{D}$ involves the zeroth order Hankel transform of $g(r)-1$, solving (11) for $\hat{c}(k)$, doing the inverse Hankel transform to get $c(r)$, and finally solving (2) to get $u / k_{B} T$.

\section{A. Effective Potentials for the Conservative Case}

Let us start showing the behavior of the calculated Effective Potentials for the conservative case, where we have set $\epsilon=1$ and $\mu=0$. This is a situation that clearly falls outside of experimental possibilities, and we are reporting it here only as a validation of 
the method, that is, of the use of the OZ-PY scheme to extract effective potentials from the simulated PDFs. In Figs. (2) and (3) we have an example of the PDFs and effective potentials for the non-dissipative granulate, with the parameters given in the figure caption. It is clear that the OZ-PY procedure manages to extract potentials that are very close to those of a hard disk fluid, even if we have to take into account that our system is not really perfectly 2D, something that is noticeable in: (a) the behavior of the PDFs for $r$ slightly smaller than $\sigma$, where a non-zero probability is found, (b) the mild hump between $\sigma$ and $2 \sigma$ found in the potential, and (c) a very narrow potential well that appears at contact. These variations with respect to the perfect hard disk potential are related to the apparent penetrability of spheres, that is, to the fact that the $2 \mathrm{D}$ projection of this $3 \mathrm{D}$ system shows distances between centers smaller than one diameter, obviously without any real interpenetration involved. This apparent overlapping could lead to the very narrow potential at contact, while the mild hump may be an artifact produced by confinement of the spheres in a narrow quasi-2D space. Still, it should be noticed that the numerical values for the variations of the potential are quite small, even more if compared to the non-conservative examples to be covered next. It should also be noticed that the potential shows some short wavelength oscillations, an unavoidable by product of the truncation in the back and forth Fourier transforms required for the solution.

\section{B. Effective Potentials as a Function of Energy Dissipation, and Forcing Reso-} nances

Going now into the dissipative case, let us begin by showing some examples of the behavior of the effective potentials found for different sets of parameters. In Fig. (4) we show the effective potentials obtained for a constant packing fraction $\phi=0.40$ for four different nondimensional accelerations $\Gamma=1.05,1.15,1.35$ and 1.45. (Some of the PDFs from where these potentials were extracted are shown latter, in Fig. (11)). As expected, and in coincidence

with the experimental results reported in [20], there is an attractive well in the effective potential, and a tendency to get more attractive potentials as the restitution coefficient is lowered. This effect can be understood as a result of the smaller distances between particles that are found after mutual inelastic collisions, as compared with those distances expected for elastic ones, something that occurs simply because the smaller separation velocities found 


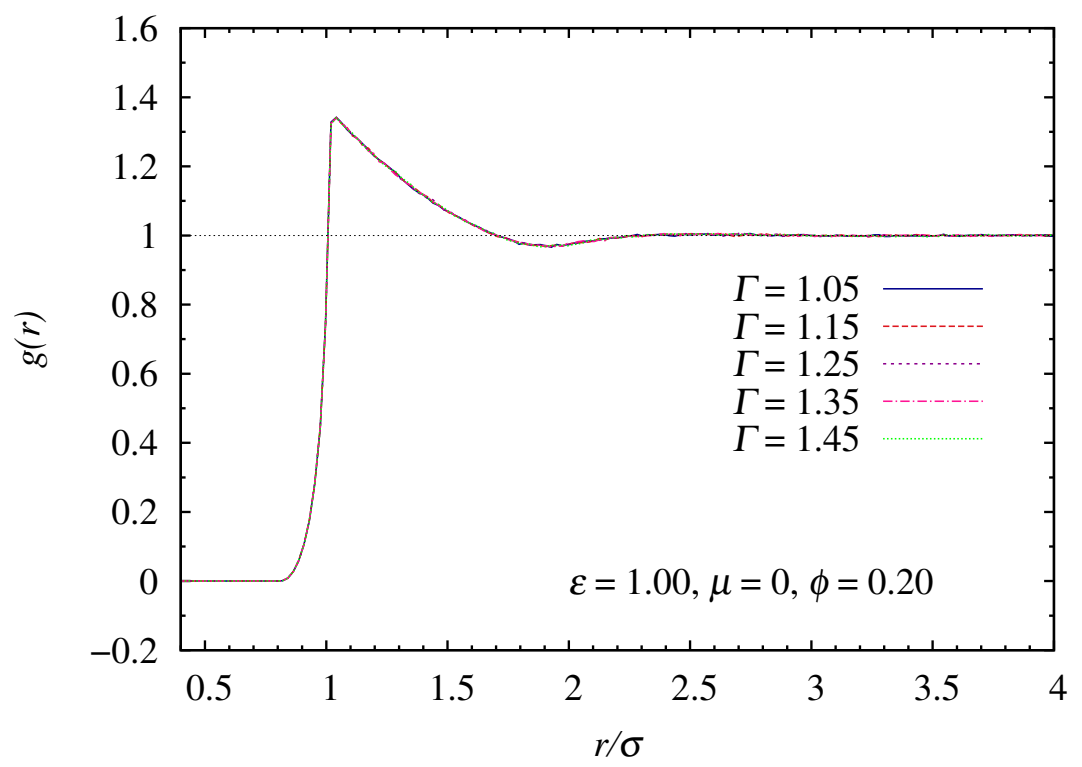

FIG. 2. Pair Distribution Function for the conservative case, $\epsilon=1, \mu=0$. Here we show the $\phi=0.2$ case, for all the $\Gamma$ values considered $(\Gamma=1.05,1.15,1.25,1.35$ and 1.45$)$. The different lines superpose almost perfectly, implying that the vibration strength is irrelevant in the confined conservative case. PDFs for larger values of $\phi$ behave in a completely similar manner, just showing some more structure close to contact.

after the contacts. In this sense we find that the PDFs for steady states of dissipative systems register, in general, an increase of the spatial correlation at contact for decreasing values of the restitution coefficient, and thus, a tendency of the effective potentials to display deeper wells for more dissipative dynamics.

There is, however, one general exceptions to this rule, within the range of parameters we have explored. An example is given in Fig. (4-b), where the deepest well actually corresponds to $\epsilon=0.80$, which is not the most dissipative value studied, and other example is given in Fig. (4-c), where the deepest well corresponds now to the most elastic case $\epsilon=0.90$. Similar anomalies appear for other combinations of parameters, although "normal" behavior always happens for very low or very high $\Gamma$ values.

It is clear then that there is no simple scaling of the effective potentials as $\epsilon$ changes: a larger restitution coefficient may be associated to a deeper potential well, or the potentials for different values of $\epsilon$ may cross each other, although keeping the general trend of more attractive potentials for smaller $\epsilon$. To understand this peculiarities, let us concentrate in the effect that different values of the forcing $\Gamma$ have in the dynamics: we find that the clustering 


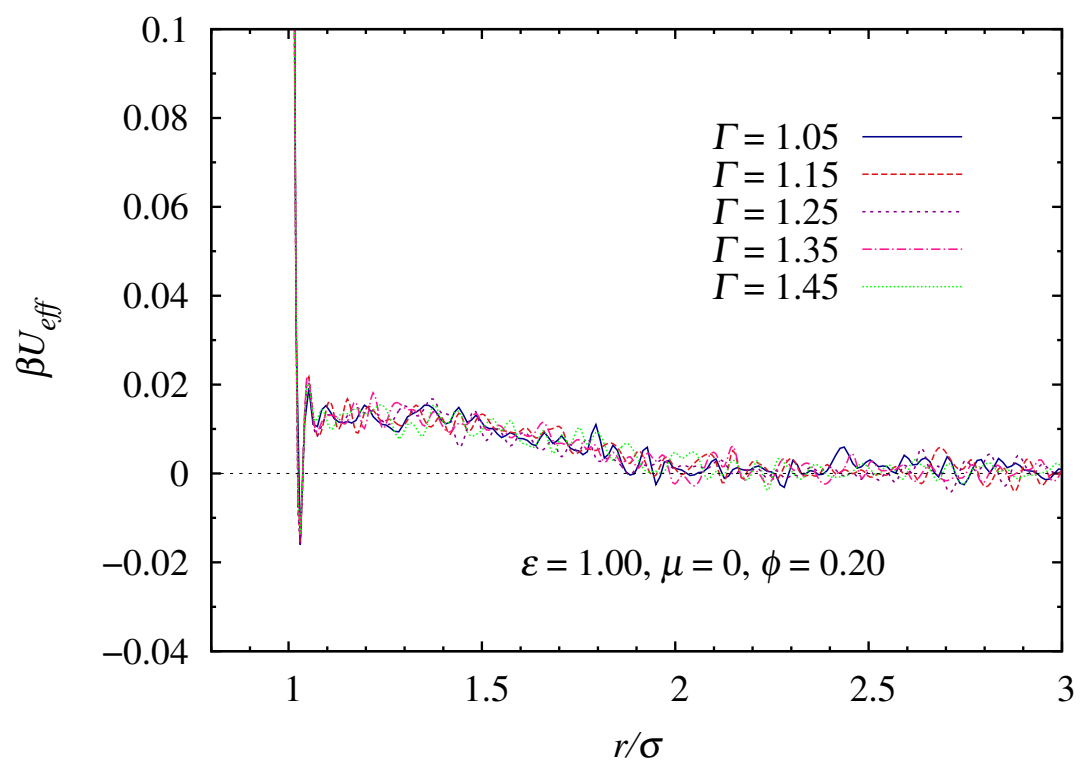

FIG. 3. Effective potentials corresponding to the PDFs given in the previous figure. The small oscillations induced by truncation in the Fourier transforms appear clearly, together with a narrow and not too deep well at contact, plus a mild potential barrier between $r / \sigma=1$ and $r / \sigma=2$. As mentioned in the text, this narrow well and the mild potential barrier are most probably artifacts due to the imperfect two-dimensional character of the granulate.

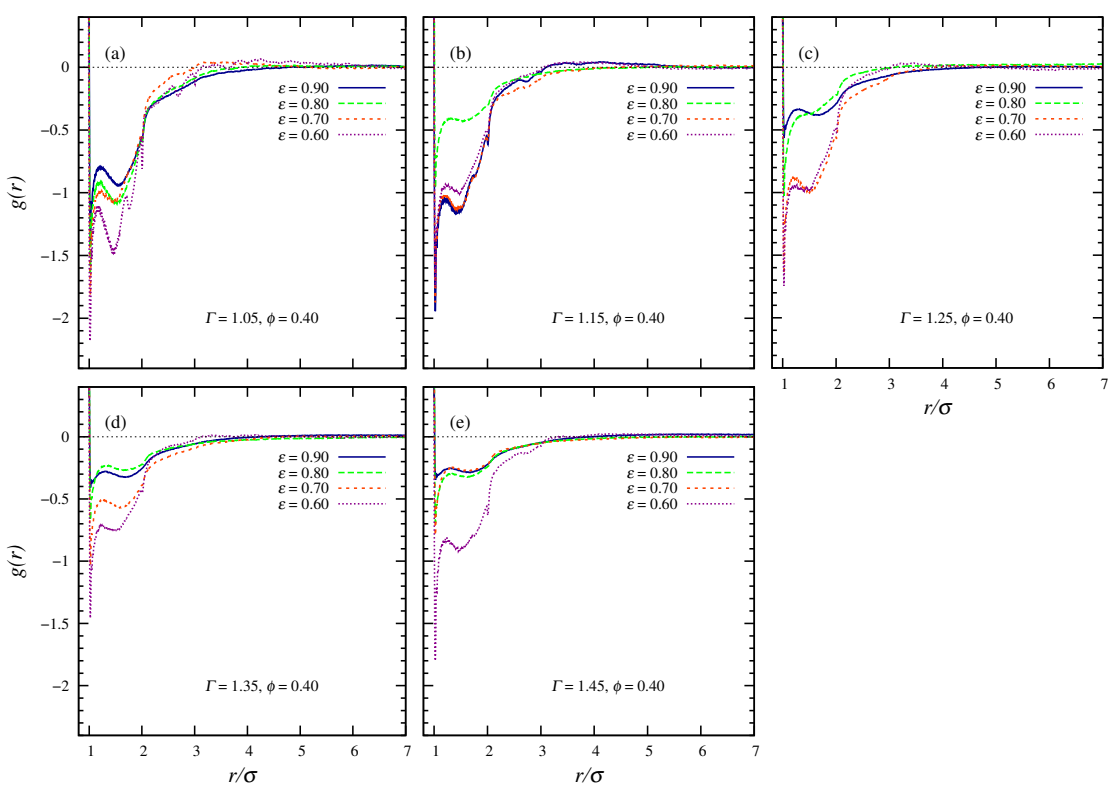

FIG. 4. Effective potentials corresponding to the PDFs obtained for $2 \mathrm{D}$ packing fraction $\phi=0.40$, at four different values of the restitution coefficient, at five different values of forcing $\Gamma$. (a): $\Gamma=1.05 ;(\mathrm{b}): \Gamma=1.15 ;(\mathrm{c}): \Gamma=1.25 ;(\mathrm{d}): \Gamma=1.35 ;(\mathrm{e}): \Gamma=1.45$. 
which is mainly responsible for the apparent increasing attraction between particles can be enhanced for a narrow range of this parameter. This is due to resonances that appear at narrow windows in forcing, whose location depend on the restitution coefficient but are independent of the packing fraction. The resonance arises from a simplification in the motion of the granulate, in the following sense: ignoring for the moment being the particle-particle collisions, the motion of these particles is just a collection of parabolic flights above the floor. In terms of vertical displacement, these parabolas may be of just one or a few types (modes), that is, of just one or a few heights, and this is what constitutes a resonance. On the contrary, the granulate may display a form of motion composed of many different modes (parable with different heights). Due to the dissipative-forced character of the motion, a mode corresponds not just to the presence of one or a few parabolic parabolic heights, but also involves synchronization, and so in the granulate we will have many particles displaying the same vertical motion. Parabolas of different heights will of course be unsynchronized.

The evidence for these two forms of motion - simple an complex, synchronous and asynchronous - , now incorporating the effects of collisions, is given in Fig. (5)), which shows the probability density for the height of the particles above the floor, $p(z)$, vs. height $z$. In this figure we can see now a single and well defined peak that appears for $\Gamma=1.14$, decreases in height and finally splits as $\Gamma$ grows, going in this way from a mode with a one-height parabola to a mode with two different parabolas. This unity in the height of the vertical motion is relevant to the horizontal behavior of the system because, among particle-particle collisions, only those that happen between particles with different heights introduce horizontal scattering, and therefore reduce plane correlations. On the contrary, for parabolic motions of the same height, collisions are perfectly lateral, and always reduce the in-plane kinetic energy.

Thus, it is necessary to consider not only the absolute value of the exciting nondimensional acceleration $\Gamma$, but also whether or not there are coincidences (resonances) in the vertical motion of the particles. These resonant phenomena have already been seen in the case of oscillons [36]. In Fig. (66) we show the snapshots of the simulated gas for $\Gamma=1.15$, $\phi=0.40$ and four different restitution coefficients $\epsilon$, corresponding to the Fig. (4-c) in which anomalous behavior is detected, in particular for the value $\epsilon=0.80$.

Another way of showing the effects of these resonances is given in Fig. (7), where correlations at contact, defined by the maximum value of the PDF, are plotted as a function of 


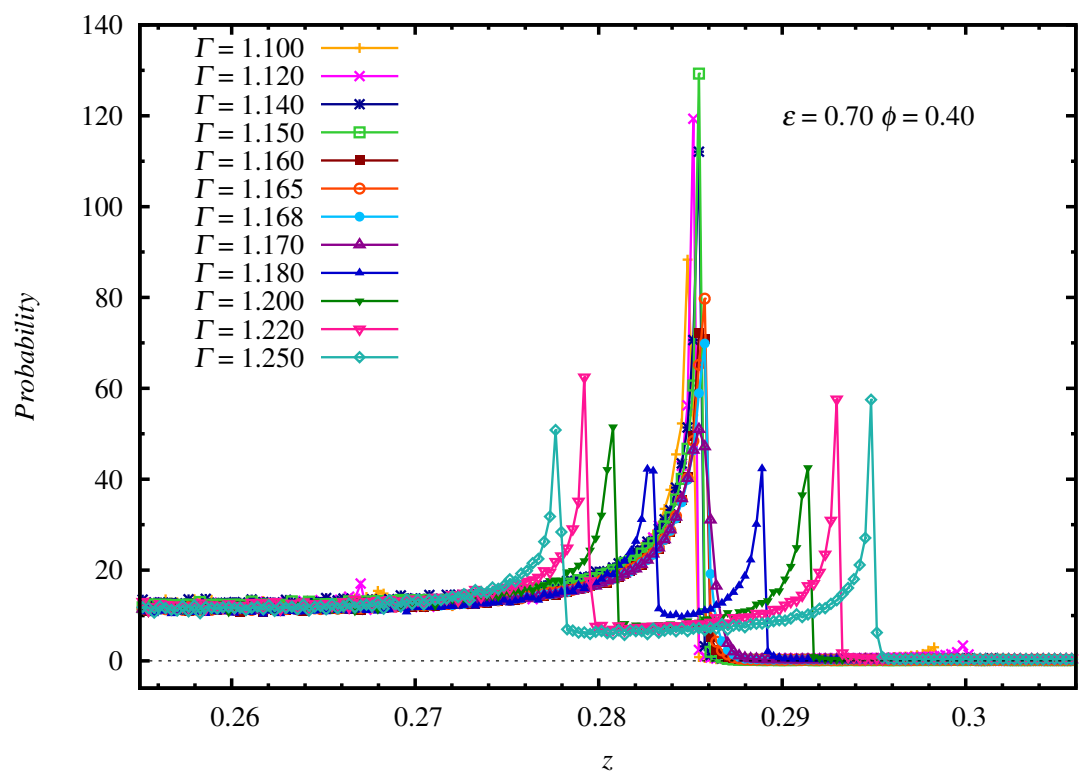

FIG. 5. Probability density for the height of the particles above the floor for 2D packing fraction $\epsilon=0.70$ and $\phi=0.40$, at several different values of $\Gamma . \Gamma=1.100 ; \Gamma=1.120 ; \Gamma=1.140 ; \Gamma=1.150 ;$ $\Gamma=1.165 ; \Gamma=1.168 ; \Gamma=1.170 ; \Gamma=1.180 ; \Gamma=1.200 ; \Gamma=1.220 ; \Gamma=1.250$.

$\Gamma$, for a fine tuned exploration of this parameter for $\epsilon=0.80$ and $\phi=0.20,0.30$ and 0.40 . There one can see that the overall response of the maximum value of $g(r)$ is to decrease with growing $\Gamma$ (growing shaking amplitude), since large shaking intensity or "granular temperature" tends to destroy correlations. If the maxima of the PDFs are plotted as a function of $\Gamma$, avoiding resonant values, they show a roughly exponential decay with $\Gamma$. Seeing that the correlation becomes very large for $\Gamma \rightarrow 1$, we try a fit $g_{M a x}(r)=g_{0}+A \exp \left((\Gamma-1) /\left(\Gamma_{0}-1\right)\right)$ For the $\phi=0.4, \epsilon=0.8$ case we get $g_{0}=3.2, A=8.8$ and $\Gamma_{0}=1.041$. This fit can be seen in the inset of Fig. (17). But, as already described, this monotonic behavior is interrupted by a narrow peak centered at $\Gamma=1.22$ showing a resonant "cooling down" of the horizontal degrees of freedom of the system. In Fig. (8) effective potential depths for $\epsilon=0.80$ and $\phi=0.20,0.30$ and 0.40 , are plotted. The monotonic decrement of the maximum interaction energy is reversed by a resonant peaking attraction in all cases.

In Fig. (9), correlations at contact as a function of $\Gamma$ are shown for different restitution coefficients at a fixed packing fraction of $\phi=0.40$. The best defined resonant peak is the one shown in Fig. (7) for $\epsilon=0.80$, while for different restitution coefficients broader peaks appear centered at lower $\Gamma$ values. Here, as in the case of an inertial-elastic systems, when dissipation increases the resonant peak broadens. 


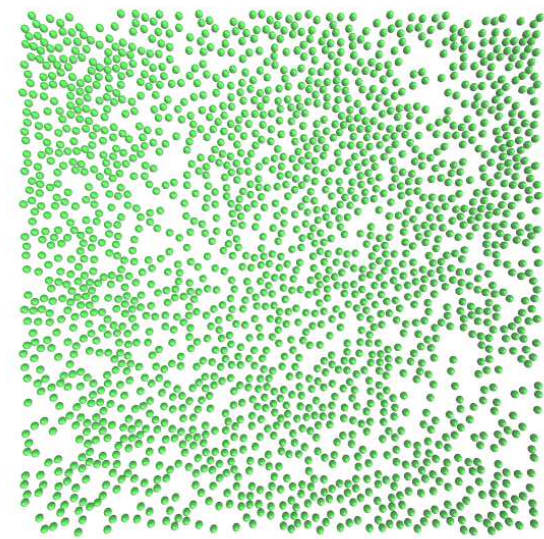

a) $\epsilon=0.60, \Gamma=1.15$ and $\phi=0.40$

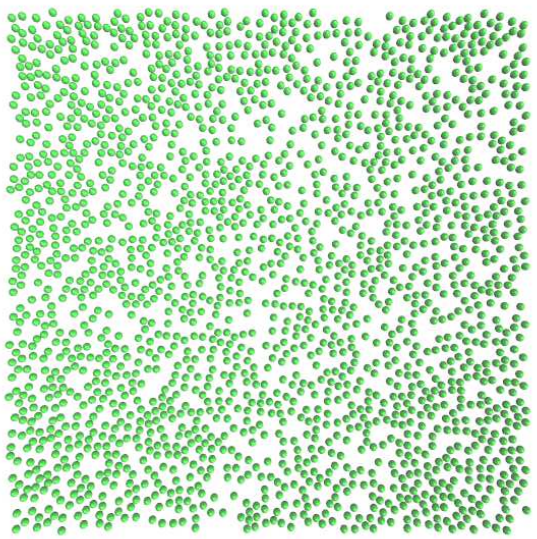

c) $\epsilon=0.80, \Gamma=1.15$ and $\phi=0.40$

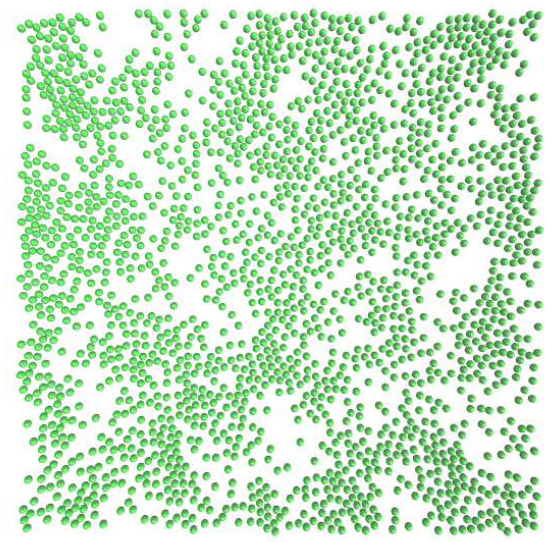

b) $\epsilon=0.70, \Gamma=1.15$ and $\phi=0.40$

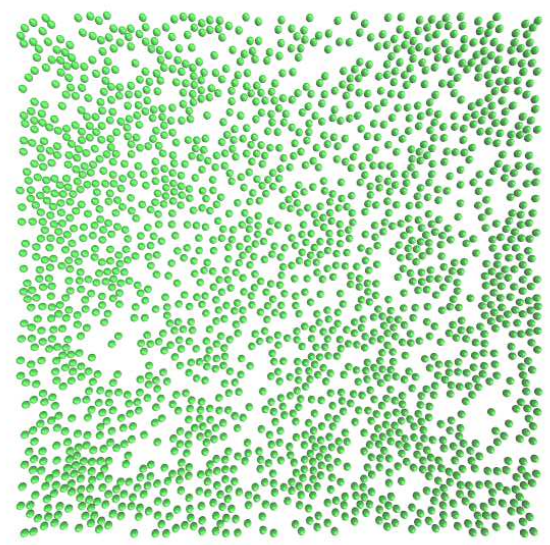

d) $\epsilon=0.90, \Gamma=1.15$ and $\phi=0.40$

FIG. 6. Snapshots of the simulated granular gas corresponding to the same conditions used in the previous Figure, showing the clustering tendency of the gas as the restitution coefficient decreases, cooling down the steady state of the granular gas.

Therefore, in general, for $\Gamma$ values out of resonance the decreasing tendency of correlation at contact with increasing shaking strength dominates, together with an increase in correlation with decreasing elasticity, as can be seen in Fig. (7).

In the supplementary material section a set of tables containing all the explored experimental conditions are presented, showing for all cases agreement with the general tendency described in the particular cases analyzed previously in the text, and in Figs. (17) and (91).

There are some other noteworthy features in the potentials found. In particular, they 


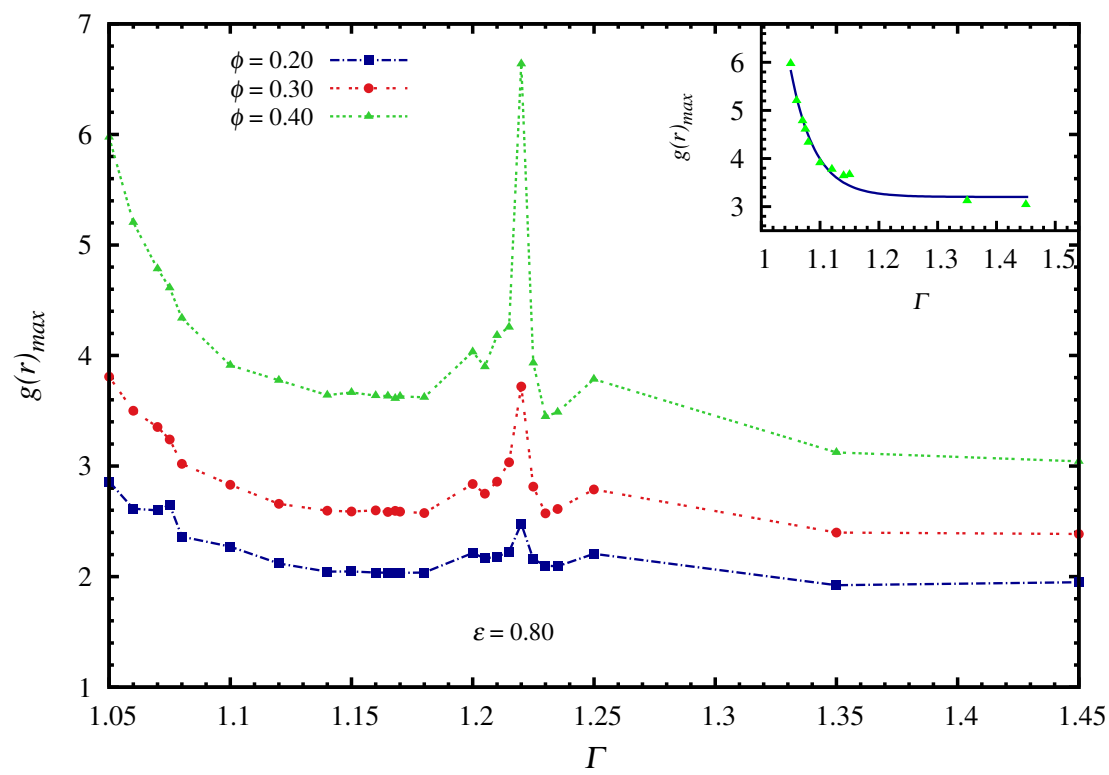

FIG. 7. Maxima of the PDFs as a function of $\Gamma$ for three different packing fractions $\phi=0.20$ (black squares), $\phi=0.30$ (red circles), and $\phi=0.40$ (green triangles). A clear resonant peak occurs at $\Gamma$ $=1.22$. In the inset the maximum of $g(r)$ is shown together with the exponential fit given in the text, omitting resonant values.

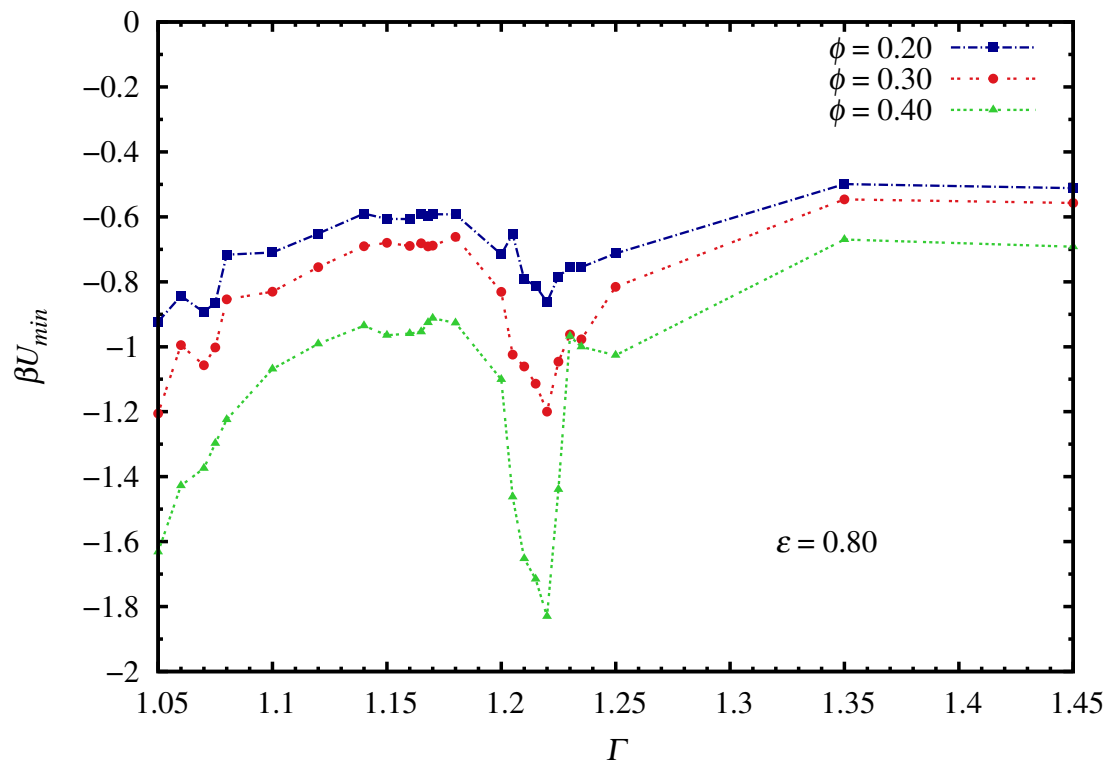

FIG. 8. Minima of pair interaction potentials as a function of $\Gamma$ for three different packing fractions $\phi=0.20$ (black squares), $\phi=0.30$ (red circles), and $\phi=0.40$ (green triangles); a clear resonant minimum occurs at $\Gamma=1.22$. 


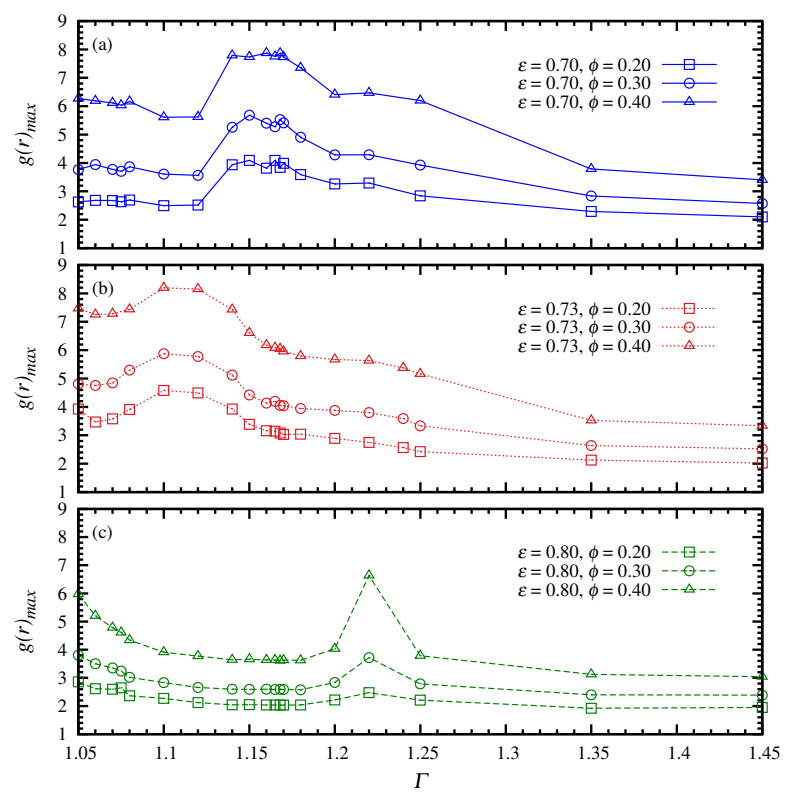

FIG. 9. Effective potentials corresponding to the PDFs obtained for $2 \mathrm{D}$ packing fraction $\phi=0.40$, at four different values of the restitution coefficient, at five different values of forcing $\Gamma$. (a): $\Gamma=1.05 ;(\mathrm{b}): \Gamma=1.15 ;(\mathrm{c}): \Gamma=1.25 ;(\mathrm{d}): \Gamma=1.35 ;(\mathrm{e}): \Gamma=1.45$.

display a kink — for small $\epsilon$, a small well — for $r / \sigma=2$, signaling the fact that the arrangements of three particles in line are specially efficient for energy dissipation; it is important to notice that no particular features are found for the next-nearest distance, $r / \sigma=\sqrt{3}$, implying that a closed-pack triangle of grains does not represent a strongly dissipative configuration. For small $\epsilon$, some of these potentials also show a wide well between $r / \sigma=1$ and $r / \sigma=2$, at a distance clearly larger than contact. These peculiarities are also found in the original experiments, as can be seen for the line denoted "painted steel" in Fig. (3) of ref. [20], which shows a wide potential well between $r / \sigma=1$ and $r / \sigma=2$; and in the line denoted "plastic" in the same figure, where a small kink at $r / \sigma=2$ can be seen. It is however clear that, given that there are some differences between the layouts for the experiment and the simulation, that there is an experimental dispersion in sizes, and also some other sources of experimental noise, one should not expect that the very detailed features found in the strongly controlled simulational environment should always be present in the experimental results. Notice finally that the secondary attractive well developed $($ at $r / \sigma=2)$ is consistent with the original finding of the inelastic collapse singularity that apparently halted the simulations of McNamara and Young [31]. 


\section{VALIDATION OF THE EFFECTIVE POTENTIALS: MONTE CARLO SIM- ULATIONS}

The basic assumption of the results presented up to now is that the stationary configurations of the forced-dissipative system studied can be understood in terms of an equilibrium 2D fluid with an effective potential that emerges, trough the dynamics, from the forcing, density and dissipative characteristics of the system. To validate this idea, we need to see if in fact the calculated potentials induce the same statics in the system. To do this we implement in this section Monte Carlo Simulations (MCS) of a corresponding 2D fluid, using a system containing the same number of disks as their corresponding EDMD experiments, affected by the effective potential obtained by EDMD; that is, the attractive interaction potential between pairs of particles as a function of interparticle distance is inserted numerically into a Monte Carlo simulation. It should be noted that a typical attractive effective interaction obtained by EDMD has a maximum range between 3 and 5 particle diameters, and beyond that it can be considered null for any practical purpose; this allows us to introduce a cut-off distance of $5 \sigma$. Initial conditions was set by randomly distributing a set of disks in the sites of an hexagonal matrix. The side of the simulation cell is chosen to satisfy the desired

packing fraction for a given number of particles (800 in all runs), using $L=\sqrt{N \pi r^{2} / \phi}$. We used in most cases 20,000 Monte Carlos sweeps. The PDFs for the granular fluid of disks were calculated, and, as can be seen in Fig. (10), they are quantitatively very similar, almost identical, to those obtained from the original molecular dynamics. This accounts for the two equivalent descriptions: on the one hand a fluid of disks in thermodynamic equilibrium interacting via a potential (representing a conservative force by definition); and on the other hand a granular gas in stationary state in which the energy lost due to inelastic collisions is injected back into the system trough sinusoidal vertical shaking. In Fig. (11) PDFs measured from the 3D-EDMS and from the effective-potential fluids in 2D Monte Carlo simulations are compared for different packing fractions, shaking accelerations, and restitution coefficients. They show excellent agreement in reproducing the pair distribution for both diluted and concentrated fluids (Fig. (11 - a) and Fig. (11 -b)), from the very elastic to the very inelastic limits (Fig. (11-c) and Fig. (11-d)), and for cold or hot gases (high or low $\Gamma$ respectively in Fig. (11- $\mathrm{e}$ ) and Fig. (11-f)). The very good agreement observed between the PDFs measured in the fully 3D EDMD of a vertically shaken granular gas in a 


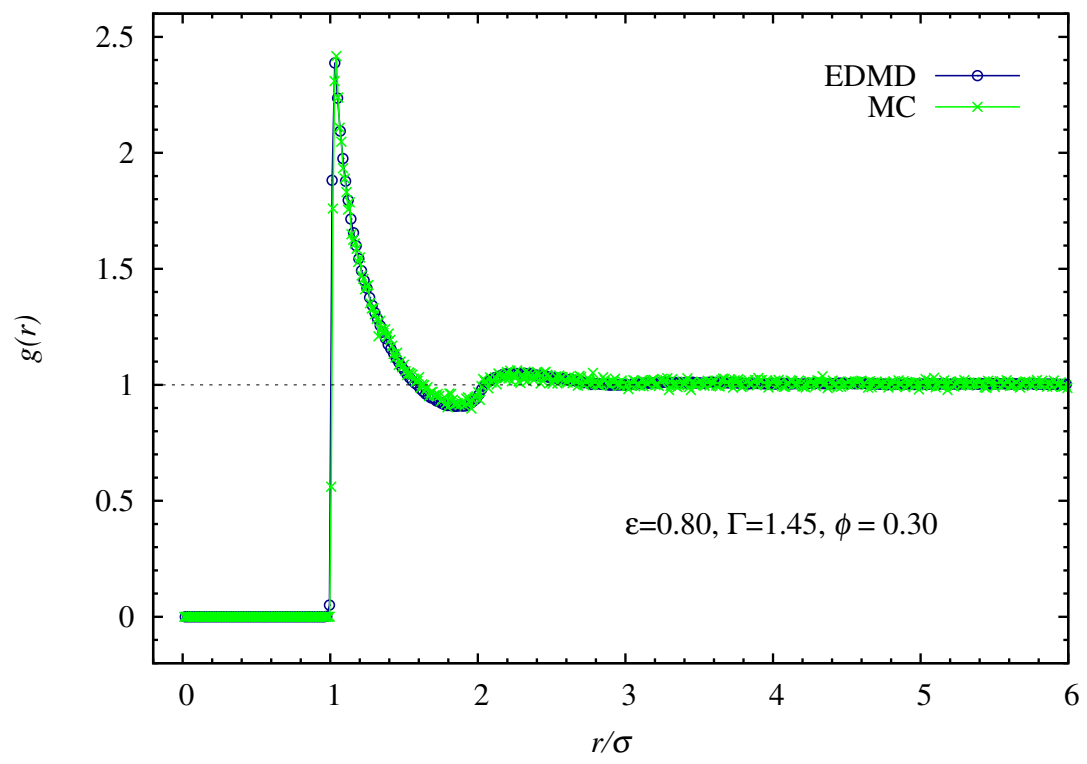

FIG. 10. Pair Distributions Functions obtained for a restitution coefficient $\epsilon=0.80$, acceleration $\Gamma=1.45$ and packing fraction $\phi=0.30$, obtained from the original EDMD (blue circles) and from a MC simulation with the derived effective potential (green crosses).

stationary out-of-equilibrium state, and those obtained using 2D Monte Carlo simulations of disks interacting trough the effective potential obtained in this way, in our opinion validates considering a granular gas excited trough shaking not as an ideal hard sphere gas but instead as a short-range interacting gas. Of course, this interacting granular gas can undergo condensation if the temperature (associated to the system energy injection rate) is lower than certain threshold value.

\section{CONCLUSIONS AND FINAL COMMENTS}

We have simulated the quasi-2D granular gas produced by sinusoidal vertical shaking of a horizontal flat substrate in which a set of spheres can bounce and roam. The simulations are carried out using a fully 3D EDMD. Two-dimensional PDFs are obtained from the projected sphere center positions, for diverse sets of parameters, and used as the input for an inversion procedure in order to get an effective pair potential that describes the apparent attractive interaction among pairs of particles. The inversion procedure involves the 2D OrnsteinZernique equation using the Percus-Yevick closure; this of course assumes as hypothesis the thermodynamic equilibrium of the system. The effective potentials thus obtained are later 


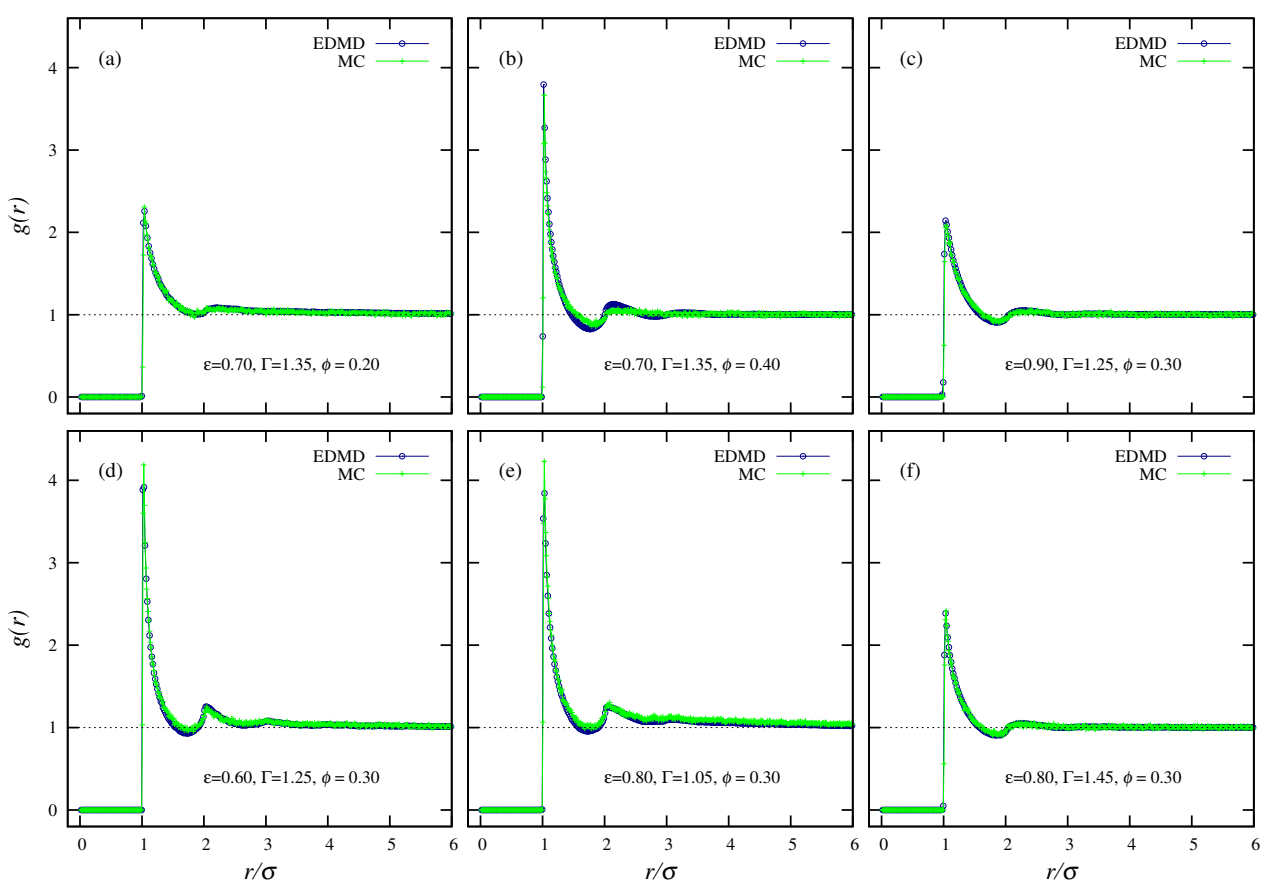

FIG. 11. Pair Distribution Functions obtained via Monte Carlo (green crosses), and EDMD (blue circles) methods. Panels (a) and (b) correspond to the minimum and maximum packing fractions used: $\phi=0.20$ and 0.40 respectively. Panels (c) and (d) correspond to the minimum and maximum restitution coefficients used: $\epsilon=0.60$ and 0.90 . Panels (e) and (f) correspond to the minimum and maximum vertical accelerations used: $\Gamma=1.05$ and 1.45 .

on used as inputs for Monte Carlo simulations of a 2D gas of disks. We have found very good agreement in the PDFs obtained from both procedures. Then, a dissipative granular gas in which energy injection maintains a steady state can be viewed as an equilibrium system of particles interacting through a potential well. The Correlation at contact grows - and consequently the effective pair interaction potential well becomes deeper - as the restitution coefficient $\epsilon$ decreases, as observed experimentally [20] and in previous simulations [19]. A fundamental exception to this trend does emerge, due to a resonant cooling down of the system that appears for the synchronization of the parabolic flights of the particles in the granulate, given the appropriate combination of shaking amplitudes and restitution coefficients.

Among the issues that remain open with respect of the effective potentials discussed here, we would like to comment on two of them. The first one is how far can we take this analogy for lower values of the forcing, where condensation appears. The results found here do show 
strong sensitivity of the effective potentials to density, and therefore it is not clear at all that, in a situation where there is coexistence between a (crystallized) condensate and mobile and more diluted fluid, the same effective potential may be used for both. There is one other well known situation where effective forces should depend on local densities, and still a single effective interaction potential manages to produce adequate phase diagrams; that is the case

of depletion interactions in bidisperse mixtures of spherical colloids [37, 38]. For the present case we still do not have an answer. A second point of interest is whether or not the high concordance obtained for the static behavior of the two considered systems, as shown in the PDFs, will also extend to dynamical quantities, such as the diffusion coefficients and Intermediate Scattering Functions. This of course will require the use of soft-disc simulations, since the definition of time scales in Monte Carlo simulations is not completely obvious. We are now exploring these possible extension of the forced-dissipative to equilibrium analogy.

\section{ACKNOWLEDGMENTS}

We acknowledge support from CONACyT though grant 221961. S. V.-P. acknowledges the support from a CONACyT Doctorate Fellowship 290611 and scholarship 290935. 
[1] J. Duran Sands, Powders, and Grains: An Introduction to the Physics of Granular Materials (Partially Ordered Systems), Springer (2000).

[2] H. M. Jaeger, S. R. Nagel and R. P. Behringer, Granular solids, liquids, and gases, Rev. Mod. Phys. 681259 (1996).

[3] Bizon, C. Shattuck, M. D. Swift, J. B. McCormick, W. D. Swinney, H. L., Patterns in 3D Vertically Oscillated Granular Layers: Simulation and Experiment, Phys. Rev. Lett. 8057 (1998).

[4] Roeller, K. Clewett, J. P. D. Bowley, R. M. Herminghaus, S. Swift, M. R., Liquid-Gas Phase Separation in Confined Vibrated Dry Granular Matter, Phys. Rev. Lett. 107048002 (2011).

[5] Tata, B. V. R. Rajamani, P. V. Chakrabarti, J. Nikolov, A. Wasan, D. T., Gas-Liquid Transition in a Two-Dimensional System of Millimeter-Sized Like-Charged Metal Balls, Phys. Rev. Lett. 843626 (2000).

[6] Nowak, E. R. Knight, J. B. Ben-Naim, E. Jaeger, H. M. Nagel, S. R., Fluctuations in the Density of a Granular Material During Vibration, Phys. Rev. E 571971 (1998).

[7] Eshuis, P. van der Weele, K. van der Meer, D. Bos, R. Lohse, D., Phase diagram of vertically shaken granular matter, Phys. Fluids 19123301 (2007).

[8] P. M. Reis, R. A. Ingale and M. D. Shattuck, Caging Dynamics in a Granular Fluid, Phys. Rev. Lett. 98188301 (2007).

[9] J. S. Olafsen and J. S. Urbach, Clustering, Order, and Collapse in a Driven Granular Monolayer, Phys. Rev. Lett. 814369 (1998).

[10] J. S. Olafsen and J. S. Urbach, Velocity distributions and density fluctuations in a granular gas, Phys. Rev. E 60 R2468 (1999).

[11] P. Melby et al., The dynamics of thin vibrated granular layers, J. Phys.: Condens. Matter 17 S2689 (2005).

[12] F. Vega-Reyes and J. S. Urbach, Effect of inelasticity on the phase transitions of a thin vibrated granular layer, Phys. Rev. E 78051301 (2008).

[13] J. S. Olafsen and J. S. Urbach, Two-Dimensional Melting Far from Equilibrium in a Granular Monolayer, Phys. Rev. Lett. 95098002 (2005).

[14] P. M. Reis, R. A. Ingale and M. D. Shattuck, Crystallization of a Quasi-Two-Dimensional 
Granular Fluid, Phys. Rev. Lett. 96258001 (2006).

[15] B. J. Alder and T. E. Wainwright, Phase Transition in Elastic Disks, Phys. Rev. 127359 (1962).

[16] Engel, M. Anderson, J. A. Glotzer, S. C. Isobe, M. Bernard, E. P. Krauth, W., Hard-disk equation of state: First-order liquid-hexatic transition in two dimensions with three simulation methods, Phys. Rev. E 87042134 (2013).

[17] X. Nie, E. Ben-Naim and S. Y. Chen, Dynamics of vibrated granular monolayers, Europhys. Lett. 51679 (2000).

[18] G. Pérez-Angel and Y. Nahmad-Molinari, Bouncing, rolling, energy flows, and cluster formation in a two-dimensional vibrated granular gas, Phys. Rev. E 84041303 (2011).

[19] J. A. Perera-Burgos, G. Pérez-Ángel and Y. Nahmad-Molinari, Diffusivity and weak clustering in a quasi-two-dimensional granular gas, Phys. Rev. E 82051305 (2010)

[20] R. A. Bordallo-Favela et al., Effective potentials of dissipative hard spheres in granular matter, Eur. Phys. J. E 28395 (2009).

[21] B. Derjaguin and L. Landau, Theory of the stability of strongly charged lyophobic sols and of the adhesion of strongly charged particles in solutions of electrolytes, Acta Phys. Chim. URSS 14633 (1941).

[22] J. E. Verwey and J. T. G. Overbeek, Theory of the stability of lyophobic Colloids, Elsevier, Amsterdam (1948).

[23] P. C. Hiemenz and M. Rjagopalan, Principles of Colloid and Surface Chemistry, 3rd edition, Marcel Dekker, New York (1997).

[24] S. Asakura and F. Oosawa, On Interaction between Two Bodies Immersed in a Solution of Macromolecules, J. Chem. Phys. 221255 (1954).

[25] S. Asakura and F. Oosawa (1958) Interaction between Particles Suspended in Solutions of Macromolecules, J. Pol. Sci. 33183 (1958).

[26] M. P. Ciamarra, A. Coniglio and M. Nicodemi, Dynamically Induced Effective Interaction in Periodically Driven Granular Mixtures, Phys. Rev. Lett. 97038001 (2006).

[27] T. Pöschel and T. Schwager, Computational Granular Dynamics: Models and Algorithms, Springer (2005).

[28] D. C. Rapaport, The Art of Molecular Dynamics Simulation, 2nd edition, Cambridge University Press (2004). 
[29] G. Perez, Numerical simulations in granular matter: The discharge of a 2D silo, Pramana J. Phys. 70989 (2008).

[30] T. Schwager, V. Becker and T. Pöschel, Coefficient of tangential restitution for viscoelastic speheres, Eur. Phys. J. E 27107 (2008).

[31] S. McNamara and W. R. Young, Dynamics of a freely evolving, two-dimensional granular medium, Phys. Rev. E 535089 (1996).

[32] S. McNamara and S. Luding, Energy flows in vibrated granular media, Phys. Rev. E 58813 (1998).

[33] S. González, D. Risso and R. Soto, Extended event driven molecular dynamics for simulating dense granular matter, Eur. Phys. J. Special Topics 179 (2009)

[34] M. E. Fisher, Correlation Functions and the Critical Region of Simple Fluids, J. Math. Phys. 5944 (1964).

[35] The PY and other closures for the OZ equation are well covered in many textbooks, see for instance: D.A. McQuarrie, Statistical Mechanics, (University Science Books, Sausalito, 2000); D.L. Goodstein, States of Matter, (Dover Publications, New York, 1985)

[36] J. M. Hill, M. J. Jennings, D. V. To and K. A. Williams, Dynamics of an elastic ball bouncing on an oscillating plane and the oscillon, Appl. Math. Model. 24715 (2000).

[37] M. Dijkstra, R. van Roij and R. Evans, Phase Behavior and Structure of Binary Hard-Sphere Mixtures. Phys. Rev. Lett. 812268 (1998).

[38] M. Dijkstra, R. van Roij and R. Evans, Direct Simulation of the Phase Behavior of Binary Hard-Sphere Mixtures: Test of the Depletion Potential Description, Phys. Rev. Lett. 82117 (1999). 